\title{
Asymmetric Synthesis of Polyfunctionalized Pyrrolidines from Sulfinimine-Derived Pyrrolidine 2-Phosphonates. Synthesis of Pyrrolidine 225C
}

\author{
Franklin A. Davis, ${ }^{*} \mathrm{He} \mathrm{Xu}$, Yongzhong Wu, and Junyi Zhang \\ Department of Chemistry, Temple University, Philadelphia, Pennsylvania 19122 \\ fdavis@temple.edu
}

\section{Experimental}

General Procedures: Column chromatography was performed on silica gel, Merck grade 60 (230-400 mesh). Analytical and preparative thin-layer chromatography was performed on precoated silica gel plates (250 and 1000 microns) purchased from Analtech Inc. TLC plates were visualized with UV, in an iodine chamber unless otherwise noted. THF, DCM, and ether were purchased from Fisher Scientific and dried through a Solvent Dispensing System. Optical rotations were measured on a PerkinElmer 341 polarimeter. IR spectra were recorded using $\mathrm{NaCl}$ plates or as $\mathrm{KBr}$ discs. ${ }^{1} \mathrm{H}$, ${ }^{13} \mathrm{C},{ }^{31} \mathrm{P}$ NMR were measured with a Bruker Avance 400 or Varian Inova 300. HRMS data were collected using a Fission ZAB HF double-focusing mass spectrometer at the Department of Chemistry, Drexel University, Philadelphia, PA, or Michigan State University Mass Spectrometry Facility, East Lansing, MI, or Washington University Resource for Biomedical and Bio-organic Mass Spectrometry, Department of Chemistry, Washington University, St. Louis, MO.

$(2 R, 5 R)-(+)-2$-(Dimethoxyphosphoryl)-3-oxo-5-phenylpyrrolidine-1-

carboxylic acid tert-butyl ester (5), $(2 R, 5 S)$ - (-)-5-butyl-2-(dimethoxyphosphoryl)-3-oxopyrrolidine-1-carboxylic acid tert-butyl ester (9), and (2R,5R)-(+)-5-tert-butyl- 2(dimethoxyphosphoryl)-3-oxo-pyrrolidine-1-carboxylic acid tert-butyl ester (10) were prepared as previously described. ${ }^{1}$

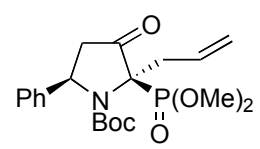

General Procedure for Horner-Wadsworth-Emmons olefination
using sodium hydride as base. Dimethyl (2R,5R)-(+)- $N$-(tert-
butoxycarbonyl)-2-allyl-3-oxo-5-phenylpyrrolidine-2- phosphonate

(6). In a 50-mL, single-necked, round-bottom flask equipped with a magnetic stirring bar, rubber septum, and argon balloon were placed (+)-5 $(0.150 \mathrm{~g}, 0.406$ $\mathrm{mmol}), \mathrm{NaH}(95 \%, 0.021 \mathrm{~g}, 0.81 \mathrm{mmol})$, and 18-crown-6 (0.227 g, $0.86 \mathrm{mmol})$ in THF $(10 \mathrm{~mL})$. The solution was stirred at $\mathrm{rt}$ for $5 \mathrm{~min}$ and allyl bromide $(0.070 \mathrm{~mL}, 0.81$ mmol) was added dropwise. After stirring for $24 \mathrm{~h}$, the reaction mixture was quenched by addition of $\mathrm{H}_{2} \mathrm{O}(3 \mathrm{~mL})$ and extracted with $\mathrm{Et}_{2} \mathrm{O}(20 \mathrm{~mL})$ and EtOAc $(2 \ldots 10 \mathrm{~mL})$. The combined organic phases were washed with brine $(3 \mathrm{~mL})$, dried $\left(\mathrm{MgSO}_{4}\right)$, and concentrated. Flash chromatography (EtOAc) afforded $0.058 \mathrm{~g} \mathrm{(35 \% )}$ of white solid; $\mathrm{mp}$ 77-78 ${ }^{\circ} \mathrm{C} ;[\alpha]^{20}{ }_{\mathrm{D}}+33.6\left(c 0.4, \mathrm{CHCl}_{3}\right)$; IR (neat) $2977,1760,1704,1253(\mathrm{P}=\mathrm{O}), 1059 \mathrm{~cm}^{-}$ ${ }^{1} ;{ }^{1} \mathrm{H} \mathrm{NMR}\left(\mathrm{CDCl}_{3}\right) \delta$ (major rotomer) $7.44(\mathrm{~d}, J=7.8 \mathrm{~Hz}, 2 \mathrm{H}), 7.24(\mathrm{~d}, J=7.5 \mathrm{~Hz}, 2 \mathrm{H})$, 7.16-7.19 (m, $1 \mathrm{H}), 5.34-5.42$ (m, $1 \mathrm{H}), 5.07-5.14$ (m, $2 \mathrm{H}), 4.74$ (br.s., $1 \mathrm{H}), 3.74-3.83$ $(\mathrm{m}, 6 \mathrm{H}), 3.41-3.47(\mathrm{~m}, 1 \mathrm{H}), 2.80-2.93(\mathrm{~m}, 3 \mathrm{H}), 1.08(\mathrm{~s}, 9 \mathrm{H}) ;{ }^{13} \mathrm{C} \mathrm{NMR}\left(\mathrm{CDCl}_{3}\right) \delta$ (major rotomer) 206.8, 153.7, 144.1, 131.1, 128.8, 127.5, 126.4, 121.8, 81.2, $71.8\left(\mathrm{~d},{ }^{1} J_{\mathrm{CP}}\right.$ $=151.1 \mathrm{~Hz}), 59.8,54.1\left(\mathrm{~d},{ }^{2} J_{\mathrm{CP}}=6.8 \mathrm{~Hz}\right), 46.6,35.0,28.2 ;{ }^{31} \mathrm{P} \mathrm{NMR}\left(\mathrm{CDCl}_{3}\right) \delta 19.49$ 
(100\%). HRMS calcd for $\mathrm{C}_{20} \mathrm{H}_{28} \mathrm{NO}_{6} \mathrm{NaP}(\mathrm{M}+\mathrm{Na})$ 432.1552. Found 432.1548.

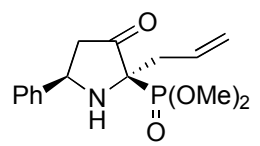

Dimeth y 1

( 2R,5R)-(+)-2-allyl-3-oxo-5-phenylpyrrolidine-2phosphonate (7). In a $25-\mathrm{mL}$, single-necked, round-bottom flask equipped with a magnetic stirring bar, rubber septum, and argon balloon was placed (+)-6 (0.040 g, $0.098 \mathrm{mmol})$ in DCM $(4 \mathrm{~mL})$. To the solution was added TFA $(0.453 \mathrm{~mL}, 0.588 \mathrm{mmol})$ at $\mathrm{rt}$ and the reaction mixture was stirred for $3 \mathrm{~h}$. At this time sat. $\mathrm{NaHCO}_{3}$ was added until $\mathrm{pH}$ reached 9 and the solution was extracted with DCM $\left(2_{-} 10 \mathrm{~mL}\right)$. The combined organic phases were washed with brine $(2 \mathrm{~mL})$, dried $\left(\mathrm{MgSO}_{4}\right)$, and concentrated. Flash chromatography (EtOAc) afforded $0.023 \mathrm{~g}(76 \%)$ of a colorless oil; $[\alpha]_{\mathrm{D}}^{20}+138.0\left(c\right.$ 0.5, $\left.\mathrm{CHCl}_{3}\right)$; IR (neat) $3299(-\mathrm{NH})$, 2954, 1752, $1243(\mathrm{P}=\mathrm{O}), 1055$ (POC) $\mathrm{cm}^{-1} ;{ }^{1} \mathrm{H}$ NMR $\left(\mathrm{CDCl}_{3}\right) \delta$ 7.42-7.43 (m, $\left.2 \mathrm{H}\right), 7.22-$ $7.41(\mathrm{~m}, 3 \mathrm{H}), 5.82-5.90(\mathrm{~m}, 1 \mathrm{H}), 5.12-5.17(\mathrm{~m}, 2 \mathrm{H}), 4.43-4.46(\mathrm{~m}, 1 \mathrm{H}), 3.81\left(\mathrm{~d},{ }^{3} J_{\mathrm{HP}}=\right.$ $10.6 \mathrm{~Hz}, 3 \mathrm{H}), 3.78\left(\mathrm{~d},{ }^{3} J_{\mathrm{HP}}=10.6 \mathrm{~Hz}, 3 \mathrm{H}\right), 2.55-2.69(\mathrm{~m}, 4 \mathrm{H}), 2.36(\mathrm{br} \mathrm{s}, 1 \mathrm{H}) ;{ }^{13} \mathrm{C}$ NMR $\left(\mathrm{CDCl}_{3}\right) \delta 210.6,142.5,131.9(\mathrm{~d}, J=9.9 \mathrm{~Hz}), 129.0,128.2,127.0,121.3,68.6(\mathrm{~d}$, $\left.{ }^{1} J_{\mathrm{CP}}=167.7 \mathrm{~Hz}\right), 57.2(\mathrm{~d}, J=13.0 \mathrm{~Hz}), 55.0\left(\mathrm{~d},{ }^{2} J_{\mathrm{CP}}=6.9 \mathrm{~Hz}\right), 54.5\left(\mathrm{~d},{ }^{2} J_{\mathrm{CP}}=7.4 \mathrm{~Hz}\right)$, 46.9, 38.3; ${ }^{31} \mathrm{P}$ NMR $\left(\mathrm{CDCl}_{3}\right) \delta$ 20.30. HRMS calcd for $\mathrm{C}_{15} \mathrm{H}_{21} \mathrm{NO}_{4} \mathrm{P}(\mathrm{M}+\mathrm{H}) 310.1208$. Found 310.1208.

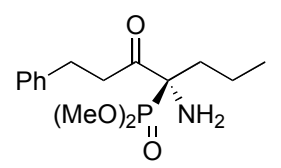

Dimethyl (R)-(-)-4-amino-3-oxo-1-phenylheptanyl-4-phosphonate (8). In a $25-\mathrm{mL}$, single-necked, round-bottom flask equipped with a magnetic stirring bar, rubber septum and $\mathrm{H}_{2}$ balloon was placed (+)-7 $(0.020 \mathrm{~g}, 0.065 \mathrm{mmol})$ and $\mathrm{Pd} / \mathrm{C}(10 \%, 0.010 \mathrm{~g})$, suspended in $\mathrm{MeOH}$ (4 mL). After stirring for $18 \mathrm{~h}$, the reaction mixture was filtered through Celite and the residue was washed with $\mathrm{MeOH}\left(2_{-} 5 \mathrm{~mL}\right)$ and $\mathrm{Et}_{2} \mathrm{O}\left(2_{-} 5 \mathrm{~mL}\right)$. The combined organic phases were dried $\left(\mathrm{MgSO}_{4}\right)$ and concentrated. Flash chromatography (EtOAc) afforded $0.019 \mathrm{~g}(95 \%)$ of a colorless oil; $[\alpha]_{\mathrm{D}}^{20}-24.9\left(c 0.4, \mathrm{CHCl}_{3}\right)$; IR (neat) 3385, $3313\left(-\mathrm{NH}_{2}\right)$, 2955, 1697, $1232(\mathrm{P}=\mathrm{O}), 1024(\mathrm{POC}) \mathrm{cm}^{-1} ;{ }^{1} \mathrm{H}$ NMR $\left(\mathrm{CDCl}_{3}\right) \delta$ 7.09-7.22 (m, $\left.5 \mathrm{H}\right), 3.71$ $\left(\mathrm{d},{ }^{3} J_{\mathrm{HP}}=10.5 \mathrm{~Hz}, 3 \mathrm{H}\right), 3.64\left(\mathrm{~d},{ }^{3} J_{\mathrm{HP}}=10.5 \mathrm{~Hz}, 3 \mathrm{H}\right), 3.09-3.15(\mathrm{~m}, 1 \mathrm{H}), 2.76-2.87(\mathrm{~m}, 3$ $\mathrm{H}), 1.44-1.81(\mathrm{~m}, 4 \mathrm{H}), 1.24-1.44(\mathrm{~m}, 1 \mathrm{H}), 0.79-0.81(\mathrm{~m}, 4 \mathrm{H}) ;{ }^{13} \mathrm{C} \mathrm{NMR}\left(\mathrm{CDCl}_{3}\right) \delta$ 206.8, 141.3, 128.9, 128.8, 126.5, $67.8\left(\mathrm{~d},{ }^{1} J_{\mathrm{CP}}=141.3 \mathrm{~Hz}\right), 54.7\left(\mathrm{~d},{ }^{2} J_{\mathrm{CP}}=7.4 \mathrm{~Hz}\right), 54.1$ $\left(\mathrm{d},{ }^{2} J_{\mathrm{CP}}=7.5 \mathrm{~Hz}\right), 40.8,36.8,30.1,16.6(\mathrm{~d}, J=11.9 \mathrm{~Hz}), 14.6, ;{ }^{31} \mathrm{P} \mathrm{NMR}\left(\mathrm{CDCl}_{3}\right) \delta$ 25.02. HRMS calcd for $\mathrm{C}_{15} \mathrm{H}_{24} \mathrm{NO}_{4} \mathrm{NaP}(\mathrm{M}+\mathrm{Na})$ 336.1341. Found 336.1340.

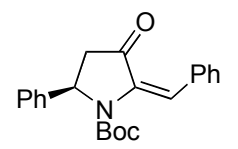

(5R)-(-)-2-Benzylidene-3-oxo-5-phenyl-pyrrolidine-1-carboxylic acid tert-butyl ester (11a). In a 100-mL, single-necked, round-bottom flask equipped with a magnetic stirring bar, rubber septum, and argon balloon was placed $\mathrm{NaH}(60 \%$ in mineral oil, $0.04 \mathrm{~g}, 1.00 \mathrm{mmol})$, which was washed with petroleum ether $(5 \mathrm{~mL})$. After removal of the petroleum ether by syringe, $\mathrm{NaH}$ was places under vacuum for $2 \mathrm{~min}$, THF $(15 \mathrm{~mL})$ was added by syringe and the solution was cooled to $0{ }^{\circ} \mathrm{C}$. In second $50 \mathrm{~mL}$ round-bottom flask was placed (+)-5 (0.25 $\mathrm{g}, 0.68 \mathrm{mmol})$ and benzaldehyde $(0.079 \mathrm{~g}, 0.745 \mathrm{mmol})$ in THF $(25 \mathrm{~mL})$, and the solution was slowly transferred via cannula to the $100 \mathrm{~mL}$ round-bottom flask. The reaction mixture was stirred at $0{ }^{\circ} \mathrm{C}$ for $1 \mathrm{~h}$ and then quenched by addition of aqueous $\mathrm{NH}_{4} \mathrm{Cl}(5$ $\mathrm{mL})$ and $\mathrm{H}_{2} \mathrm{O}(5 \mathrm{~mL})$. The solution was extracted with $\mathrm{Et}_{2} \mathrm{O}(20 \mathrm{~mL})$ and EtOAc $(2 \times 20$ $\mathrm{mL})$. The combined organic phases were washed with brine $(5 \mathrm{~mL})$, dried $\left(\mathrm{Na}_{2} \mathrm{SO}_{4}\right)$, and 
concentrated. Flash chromatography (hexanes:EtOAc, 1:1) gave $0.22 \mathrm{~g}(93 \%)$ of a yellow oil; $[\alpha]_{\mathrm{D}}{ }^{20}-26.1\left(\mathrm{c} 0.8, \mathrm{CHCl}_{3}\right)$; IR (neat) $3457 \mathrm{~cm}^{-1} ;{ }^{1} \mathrm{H} \mathrm{NMR}\left(\mathrm{CDCl}_{3}\right)-7.68$ (s, $1 \mathrm{H}), 7.52(\mathrm{~d}, J=7.5 \mathrm{~Hz}, 2 \mathrm{H}), 7.20(\mathrm{~m}, 8 \mathrm{H}), 5.31(\mathrm{~d}, J=10.0 \mathrm{~Hz}, 1 \mathrm{H}), 3.01(\mathrm{dd}, J=$ $18.4,10.1 \mathrm{~Hz}, 1 \mathrm{H}), 2.41(\mathrm{dd}, J=18.4,2.1 \mathrm{~Hz}, 1 \mathrm{H}), 1.27(\mathrm{~s}, 9 \mathrm{H}) ;{ }^{13} \mathrm{C}$ NMR $\left(\mathrm{CDCl}_{3}\right)$ 198.0, 152.8, 143.6, 134.5, 132.8, 130.5, 129.2, 128.3, 128.1, 128.0, 125.7, 122.0, 82.2, 57.9, 44.5, 28.4. HRMS calcd for $\mathrm{C}_{22} \mathrm{H}_{23} \mathrm{NO}_{3} \mathrm{Na}(\mathrm{M}+\mathrm{Na})$ 372.1576. Found 372.1583.

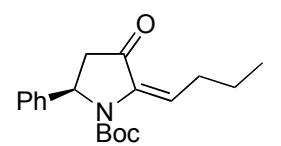

General Procedure for Horner-Wadsworth-Emmons olefination using DBU and LiCl. (5R)-(+)-2-Butylidene-3-oxo-5-phenylpyrrolidine-1-carboxylic acid tert-butyl ester (11b). In a 100-mL, single-necked, round-bottom flask equipped with a magnetic stirring bar, rubber septum, and argon balloon was placed (+)-5 (0.338 g, $0.9151 \mathrm{mmol})$ and $\mathrm{LiCl}$ $(0.078 \mathrm{~g}, 1.83 \mathrm{mmol})$ in $\mathrm{CH}_{3} \mathrm{CN}(60 \mathrm{~mL})$. To the solution was added $\mathrm{DBU}(0.150 \mathrm{~mL}$, $1.00 \mathrm{mmol})$ followed by $n$-butyraldehyde $(0.200 \mathrm{~mL}, 2.29 \mathrm{mmol})$ at $0{ }^{\circ} \mathrm{C}$. After stirring at $\mathrm{rt}$ for $16 \mathrm{~h}$, the solution was quenched by addition of aqueous $\mathrm{NH}_{4} \mathrm{Cl}(20 \mathrm{~mL})$ and the solution was extracted with EtOAc $(3 \times 20 \mathrm{~mL})$. The combined organic phases were washed with brine $(20 \mathrm{~mL})$, dried $\left(\mathrm{Na}_{2} \mathrm{SO}_{4}\right)$, and concentrated. Flash chromatography afforded $0.20 \mathrm{~g}(70 \%)$ of a colorless oil; $[\alpha]_{\mathrm{D}}^{20}+34.3\left(c 3.85, \mathrm{CHCl}_{3}\right)$; IR (neat) 3519, 2968, 2873, 1769, 1715, 1369, $1158 \mathrm{~cm}^{-1}$; ${ }^{1} \mathrm{H} \mathrm{NMR}\left(\mathrm{CDCl}_{3}\right) \quad 7.24$ (m, $\left.5 \mathrm{H}\right), 6.79$ (br s, $1 \mathrm{H}), 5.30(\mathrm{~d}, J=9.5 \mathrm{~Hz}, 1 \mathrm{H}), 3.05(\mathrm{dd}, J=18.5,10.0 \mathrm{~Hz}, 1 \mathrm{H}), 2.75(\mathrm{dd}, J=15.0,8.0$ $\mathrm{Hz}, 2 \mathrm{H}), 2.47(\mathrm{dd}, 18.5,2.5 \mathrm{~Hz}, 1 \mathrm{H}), 1.52(\mathrm{dd}, J=14.8,7.8 \mathrm{~Hz}, 2 \mathrm{H}), 0.98(\mathrm{t}, J=7.5$ $\mathrm{Hz}, 3 \mathrm{H}) ;{ }^{13} \mathrm{C} \mathrm{NMR}\left(\mathrm{CDCl}_{3}\right)$ 200.0, 152.6, 143.6, 132.3, 128.8, 127.5, 125.5, 125.3, 81.3, 57.1, 44.4, 28.2, 28.1, 23.3, 14.0. Slow decomposition of $\mathbf{1 1 b}$ over several hours precluded a satisfactory HRMS.

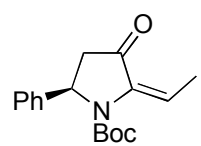

(5R)-(+)-2-Ethylidene-3-oxo-5-phenyl-pyrrolidine-1-carboxylic acid tert-butyl ester (11c). Yield: $70 \% ;[\alpha]_{D}^{20}+28.2\left(c 1.0, \mathrm{CHCl}_{3}\right)$; IR (neat) $3519,2968,2873,1769,1715,1369,1158 \mathrm{~cm}^{-1} ;{ }^{1} \mathrm{H}$ NMR $\left(\mathrm{CDCl}_{3}\right)_{-} 7.29$ $(\mathrm{m}, 3 \mathrm{H}), 7.14(\mathrm{~m}, 2 \mathrm{H}), 6.86(\mathrm{~m}, 1 \mathrm{H}), 5.28(\mathrm{~d}, J=10.4 \mathrm{~Hz}, 1 \mathrm{H}), 3.06(\mathrm{~m}$, $1 \mathrm{H}), 2.47(\mathrm{~m}, 1 \mathrm{H}), 2.22(\mathrm{~d}, J=7.6 \mathrm{~Hz}, 2 \mathrm{H}), 1.30(\mathrm{~s}, 9 \mathrm{H}) ;{ }^{13} \mathrm{C} \mathrm{NMR}\left(\mathrm{CDCl}_{3}\right) \_200.4$, $143.9,133.2,129.1,127.8,125.7,120.0,81.6,57.4,44.7,28.4,12.7$. Slow decomposition of 11c over several hours precluded a satisfactory HRMS.

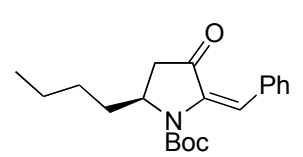

(5R)-(-)-2-Benzylidene-5-butyl-3-oxo-pyrrolidine-1-carboxylic acid tert-butyl ester (12a). Yield: 66\%; $[\alpha]^{20}-25.8\left(c 2.5, \mathrm{CHCl}_{3}\right)$; IR (neat) 3498, 2876, 1789, 1690, $1169 \mathrm{~cm}^{-1} ;{ }^{1} \mathrm{H}$ NMR $\left(\mathrm{CDCl}_{3}\right)_{-} 7.49$ $(\mathrm{m}, 3 \mathrm{H}), 7.21(\mathrm{~m}, 3 \mathrm{H}), 4.32(\mathrm{~m}, 1 \mathrm{H}), 2.68(\mathrm{~m}, 1 \mathrm{H}), 2.21(\mathrm{~m}, 1 \mathrm{H})$, $1.67(\mathrm{~m}, 1 \mathrm{H}), 1.48(\mathrm{~s}, 9 \mathrm{H}), 1.42(\mathrm{~m}, 1 \mathrm{H}), 1.23(\mathrm{~m}, 6 \mathrm{H}), 0.84(\mathrm{t}, J=9.2 \mathrm{~Hz}, 3 \mathrm{H}) ;{ }^{13} \mathrm{C}$ $\operatorname{NMR}\left(\mathrm{CDCl}_{3}\right) \quad$ 198.9, 152.9, 134.5, 132.1, 130.3, 128.2, 127.9, 122.8, 81.8, 54.5, 41.4, $36.7,28.7,27.7,22.8,14.3$. Slow decomposition of $\mathbf{1 2 a}$ over several hours precluded a satisfactory HRMS.

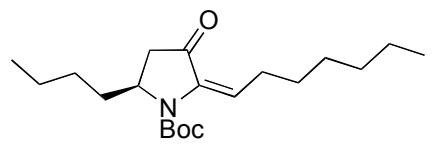

(5R)-(-)-5-Butyl-2-heptylidene-3-oxo-pyrrolidine-1carboxylic acid tert-butyl ester (12b). Yield: $75 \% ;[\alpha]^{20}{ }_{D}^{-}$ 9.7 (c 1.5, $\mathrm{CHCl}_{3}$ ); IR (neat) 3410, 2902, 1720, 1680, 1178 $\mathrm{cm}^{-1} ;{ }^{1} \mathrm{H}$ NMR $\left(\mathrm{CDCl}_{3}\right)_{-} 6.54(\mathrm{br}, 1 \mathrm{H}), 4.28(\mathrm{~m}, 1 \mathrm{H}), 2.68$ 
(m, $2 \mathrm{H}), 2.23(\mathrm{~m}, 1 \mathrm{H}), 1.67(\mathrm{~m}, 1 \mathrm{H}), 1.51(\mathrm{~s}, 9 \mathrm{H}), 1.46-1.16(\mathrm{~m}, 14 \mathrm{H}), 0.86(\mathrm{~m}, 6 \mathrm{H})$; ${ }^{13} \mathrm{C}$ NMR $\left(\mathrm{CDCl}_{3}\right)_{-} 201.5,153.0,131.7,127.0,81.3,54.0,41.5,35.5,32.2,32.0,30.3$, $30.0,28.7,27.7,26.6,22.9,22.8,14.4,14.3$. Slow decomposition of $\mathbf{1 2 b}$ over several hours precluded a satisfactory HRMS.

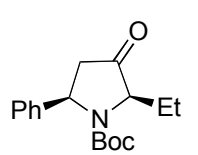

General procedure for hydrogenation. $(2 R, 5 R)-(+)-2-E t h y l-3-$ oxo-5phenyl-pyrrolidine-1-carboxylic acid tert-butyl ester (13). In a 100-mL, single-necked, round-bottom flask equipped with a magnetic stirring bar, rubber septum and $\mathrm{H}_{2}$ filled balloon was placed (+)-11c $(0.500 \mathrm{~g}, 1.73$ $\mathrm{mmol})$ and $\mathrm{Pd} / \mathrm{C}(20 \%, 0.4 \mathrm{~g})$ suspended in $\mathrm{MeOH}(20 \mathrm{~mL})$. After stirring for $4 \mathrm{~h}$, the reaction mixture was filtered through Celite and the residue was washed with $\mathrm{MeOH}(2 \times$ $20 \mathrm{~mL}), \mathrm{Et}_{2} \mathrm{O}(2 \times 20 \mathrm{~mL})$, and the combined organic phases were concentrated. Flash Chromatography (hexanes:EtOAc, 90:10) afforded $0.42 \mathrm{~g}(84 \%)$ of a white foam: $[\alpha]^{20}$ +1.0 (c 1.0, $\left.\mathrm{CHCl}_{3}\right)$; IR (neat) 3576, 1780, 1660, $1167 \mathrm{~cm}^{-1} ;{ }^{1} \mathrm{H} \mathrm{NMR}\left(\mathrm{CDCl}_{3}\right)-7.26(\mathrm{~m}$, $2 \mathrm{H}), 7.17(\mathrm{~m}, 3 \mathrm{H}), 5.19(\mathrm{~m}, 1 \mathrm{H}), 4.26(\mathrm{~m}, 1 \mathrm{H}), 2.89$ (m, $1 \mathrm{H}), 2.80(\mathrm{~m}, 1 \mathrm{H}), 1.83(\mathrm{~m}, 1$ $\mathrm{H}), 1.51(\mathrm{~m}, 1 \mathrm{H}), 1.43(\mathrm{~s}, 9 \mathrm{H}), 0.81(\mathrm{t}, J=7.6 \mathrm{~Hz}, 3 \mathrm{H}) ;{ }^{13} \mathrm{C} \mathrm{NMR}\left(\mathrm{CDCl}_{3}\right)_{-} 208.6$, 158.1 141.2, 128.8, 128.6, 126.5, 80.1, 60.7, 41.7, 29.9, 28.7, 25.0, 9.6. HRMS calcd for $\mathrm{C}_{17} \mathrm{H}_{23} \mathrm{NO}_{3}$ (M) 289.1678. Found 289.1695.

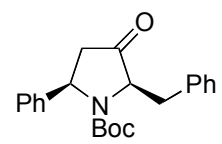

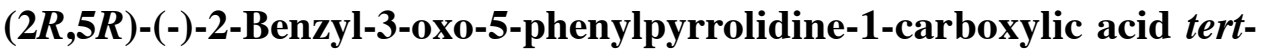
butyl ester (14). Flash chromatography (EtOAc:hexane, 3:7) afforded $0.063 \mathrm{~g}(63 \%)$ of a colorless oil; $[\alpha]_{\mathrm{D}}^{20}-24.8\left(c 0.3, \mathrm{CHCl}_{3}\right)$; IR (neat) 2975, 1757, $1694 \mathrm{~cm}^{-1} ;{ }^{1} \mathrm{H} \mathrm{NMR}\left(\mathrm{CDCl}_{3}\right) \delta$ 7.20-7.22 (m, $\left.3 \mathrm{H}\right), 7.07$ (br.s., $5 \mathrm{H}), 6.59$ (br.s., $2 \mathrm{H}), 4.87-5.09$ (m, $1 \mathrm{H}), 4.18$ (dd, $J=3.8 \mathrm{~Hz}, J=7.0 \mathrm{~Hz}, 1 \mathrm{H}), 3.23-$ 3.39 (m, $1 \mathrm{H}), 3.08-3.23$ (m, $1 \mathrm{H}), 2.89$ (dd, $J=9.2 \mathrm{~Hz}, J=19.2 \mathrm{~Hz}, 1 \mathrm{H}), 2.22-2.28$ (m, $1 \mathrm{H}), 1.22$ (br.s., $9 \mathrm{H}) ;{ }^{13} \mathrm{C} \mathrm{NMR}\left(\mathrm{CDCl}_{3}\right) \delta 212.6$, 155.7, 143.7, 137.3, 130.6, 128.9, 128.7, 127.4, 127.3, 126.5, 81.5, 65.8, 58.1, 46.1, 38.1, 28.5. HRMS calcd for $\mathrm{C}_{22} \mathrm{H}_{25} \mathrm{NO}_{3} \mathrm{Na}(\mathrm{M}+\mathrm{Na})$ 374.1732. Found 374.1727.

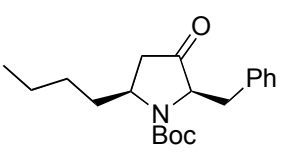

(2R,5S)-(-)-2-Benzyl-5-butyl-3-oxo-pyrrolidine-1-carboxylic acid tert-butyl ester (15). Yield: 84\%; $[\alpha]_{\mathrm{D}}^{20}-101.0\left(c\right.$ 2.0, $\left.\mathrm{CHCl}_{3}\right)$; IR (neat) 3487, 3398, 1790, $1678 \mathrm{~cm}^{-1}$; ${ }^{1} \mathrm{H} \mathrm{NMR}\left(\mathrm{CDCl}_{3}\right)-7.22(\mathrm{~m}, 3$ $\mathrm{H}), 7.08(\mathrm{~m}, 2 \mathrm{H}), 4.19(\mathrm{~m}, 1 \mathrm{H}), 4.02(\mathrm{~m}, 1 \mathrm{H}), 3.16(\mathrm{~m}, 2 \mathrm{H}), 2.65$ $(\mathrm{m}, 1 \mathrm{H}), 1.93(\mathrm{~m}, 1 \mathrm{H}), 1.49(\mathrm{~s}, 9 \mathrm{H}), 1.06(\mathrm{~m}, 6 \mathrm{H}), 0.76(\mathrm{t}, J=9.2 \mathrm{~Hz}, 3 \mathrm{H}) ;{ }^{13} \mathrm{C} \mathrm{NMR}$ $\left(\mathrm{CDCl}_{3}\right)_{-}$213.8, 155.0, 137.4, 130.7, 128.6, 127.1, 80.5, 64.8, 54.3, 42.7, 37.0, 28.8, 28.6, 22.6, 14.2. HRMS calcd for $\mathrm{C}_{20} \mathrm{H}_{30} \mathrm{NO}_{3}(\mathrm{M}+\mathrm{H})$ 332.2226. Found 332.2227.

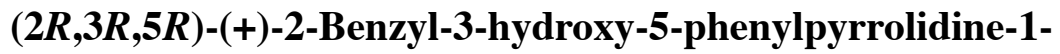

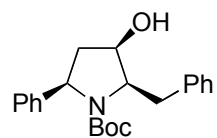
carboxylic acid tert-butyl ester (16). In a 25-mL, single-necked, roundbottom flask equipped with a magnetic stirring bar, rubber septum, and argon balloon were placed (-)-14 $(0.021 \mathrm{~g}, 0.06 \mathrm{mmol})$ and cerium(III) chloride heptahydrate $(0.089 \mathrm{~g}, 0.24 \mathrm{mmol})$ in $\mathrm{MeOH}(3 \mathrm{~mL})$. After stirring for $30 \mathrm{~min}$ at $\mathrm{rt}$ the reaction mixture was cooled to $0{ }^{\circ} \mathrm{C}$ and $\mathrm{NaBH}_{4}(0.009 \mathrm{~g}, 0.24 \mathrm{mmol})$ was added in one portion and the reaction mixture was stirred at $0{ }^{\circ} \mathrm{C}$ for $2 \mathrm{~h}$. At this time the solution was quenched with $\mathrm{H}_{2} \mathrm{O}(1 \mathrm{~mL})$, extracted with $\mathrm{Et}_{2} \mathrm{O}(5 \mathrm{~mL})$ and EtOAc $(2 \times 10 \mathrm{~mL})$, the combined organic phases were washed with brine $(2 \mathrm{~mL})$, dried $\left(\mathrm{MgSO}_{4}\right)$, and 
concentrated. Flash chromatography (EtOAc:hexane, 3:7) afforded $0.017 \mathrm{~g}(80 \%)$ of a colorless oil; $[\alpha]^{20}{ }_{D}+49.3\left(c 0.7, \mathrm{CHCl}_{3}\right)$; IR (neat) $3431(-\mathrm{OH}), 3029,2973,1669 \mathrm{~cm}^{-1} ;{ }^{1} \mathrm{H}$ $\operatorname{NMR}\left(\mathrm{CDCl}_{3}\right) \delta$ 7.19-7.40 (m, $\left.10 \mathrm{H}\right), 4.84(\mathrm{br} \mathrm{s}, 1 \mathrm{H}), 4.35(\mathrm{~d}, J=6.5 \mathrm{~Hz}, 1 \mathrm{H}), 4.25$ (br s, $1 \mathrm{H}), 3.37(\mathrm{br} \mathrm{s}, 1 \mathrm{H}), 3.11-3.16(\mathrm{~m}, 1 \mathrm{H}), 2.48-2.54(\mathrm{~m}, 1 \mathrm{H}), 2.06-2.11(\mathrm{~m}, 1 \mathrm{H}), 1.48$ $(\mathrm{m}, 1 \mathrm{H}), 1.24(\mathrm{br} \mathrm{s}, 9 \mathrm{H}) ;{ }^{13} \mathrm{C} \mathrm{NMR}\left(\mathrm{CDCl}_{3}\right) \delta 155.5,144.3,139.9,130.0,128.8(2 \mathrm{C})$, 127.1, 126.5, 125.9, 80.3, 72.0, 64.1, 60.4, 41.9, 36.5, 28.6. HRMS calcd for $\mathrm{C}_{22} \mathrm{H}_{27} \mathrm{NO}_{3} \mathrm{Na}(\mathrm{M}+\mathrm{Na})$ 376.1888. Found 376.1884.

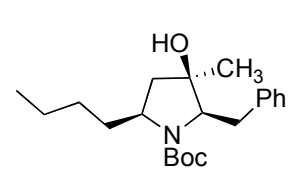

$(2 R, 3 R, 5 S)$-2-Benzyl-5-butyl-3-hydroxy-3-methyl pyrrolidine-1carboxylate (17). In a $10-\mathrm{mL}$, single-necked, round-bottom flask equipped with a magnetic stirring bar, rubber septum, and argon balloon was placed (-)-15 (0.024 g, $0.0724 \mathrm{mmol})$ in THF $(1 \mathrm{~mL})$ and ether $(1 \mathrm{~mL})$. The solution was cooled to $-78{ }^{\circ} \mathrm{C}$ and $\operatorname{MeMgBr}(72.4 \mathrm{~mL}, 3 \mathrm{M}$ in ether) was added via syringe. The reaction mixture was stirred for $1 \mathrm{~h}$ at $\mathrm{rt}$ and quenched by addition of sat. $\mathrm{NH}_{4} \mathrm{Cl}$ solution $(2 \mathrm{~mL})$. The solution was extracted with EtOAc $(3 \times 5$ $\mathrm{mL})$, the combined organic phase were washed with brine $(2 \mathrm{~mL})$, dried $\left(\mathrm{Na}_{2} \mathrm{SO}_{4}\right)$, and concentrated. Flash Chromatography (EtOAc) gave $0.016 \mathrm{~g}(65 \%)$ of a colorless oil; $[\alpha]^{20}{ }_{\mathrm{D}}+65.5\left(\mathrm{c} 1.1, \mathrm{CHCl}_{3}\right)$; IR (neat) $3450,3398,1790, \mathrm{~cm}^{-1} ;{ }^{1} \mathrm{H}$ NMR $\left(\mathrm{CDCl}_{3}\right)$ _ 7.34$7.17(\mathrm{~m}, 5 \mathrm{H}), 3.83(\mathrm{~m}, 1 \mathrm{H}), 3.65(\mathrm{~m}, 1 \mathrm{H}), 3.00(\mathrm{~m}, 1 \mathrm{H}), 2.80(\mathrm{~m}, 1 \mathrm{H}), 2.12(\mathrm{~m}, 1 \mathrm{H})$, $2.02(\mathrm{~m}, 1 \mathrm{H}), 1.83(\mathrm{~m}, 1 \mathrm{H}), 1.47-1.23(\mathrm{~m}, 9 \mathrm{H}), 1.21(\mathrm{~s}, 3 \mathrm{H}), 0.92(\mathrm{t}, J=7.2 \mathrm{~Hz}, 3 \mathrm{H})$; ${ }^{13} \mathrm{C}$ NMR $\left(\mathrm{CDCl}_{3}\right)$ - 155.4, 140.1, 130.1, 128.6, 126.3, 79.5, 77.5, 68.2, 56.7, 44.2, 38.3, 36.8, 29.3, 29.0, 28.7, 23.0, 14.4. HRMS calcd for $\mathrm{C}_{21} \mathrm{H}_{34} \mathrm{NO}_{3}(\mathrm{M}+\mathrm{H})$ 348.2539. Found 348.2540 .

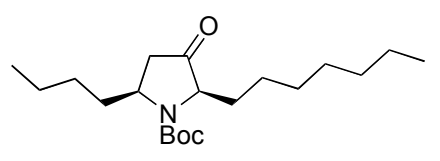

(2R,5S)-(-)-5-Butyl-2-heptyl-3-oxo-pyrrolidine-1carboxylic acid tert-butyl ester (18). In a $100-\mathrm{mL}$, singlenecked, round-bottom flask equipped with a magnetic stirring bar, rubber septum and $\mathrm{H}_{2}$ balloon was placed (-)-12b (0.435 $\mathrm{g}, 1.29 \mathrm{mmol})$ and $\mathrm{Pd} / \mathrm{C}(10 \%, 0.4 \mathrm{~g})$, suspended in $\mathrm{MeOH}(15 \mathrm{~mL})$. After stirring for 4 $\mathrm{h}$, the reaction mixture was filtered and the residue was washed with $\mathrm{MeOH}(2 \times 20 \mathrm{~mL})$, $\mathrm{Et}_{2} \mathrm{O}(2 \times 20 \mathrm{~mL})$, and the combined organic phases were concentrated. Chromatography (hexanes:EtOAc, 90:10) afforded $0.3665 \mathrm{~g}(84 \%)$ of an oil; $[\alpha]^{20}{ }_{\mathrm{D}}-41.2\left(c 1.0, \mathrm{CHCl}_{3}\right)$; IR (neat) 3423, 1760, 1650, $1155 \mathrm{~cm}^{-1} ;{ }^{1} \mathrm{H}$ NMR $\left(\mathrm{CDCl}_{3}\right) \_4.20(\mathrm{~m}, 1 \mathrm{H}), 3.90(\mathrm{~m}, 1 \mathrm{H})$, $2.72(\mathrm{~m}, 1 \mathrm{H}), 2.23(\mathrm{~m}, 1 \mathrm{H}), 1.70(\mathrm{~m}, 2 \mathrm{H}), 1.47(\mathrm{~s}, 9 \mathrm{H}), 1.27(\mathrm{~m}, 16 \mathrm{H}), 0.87(\mathrm{~m}, 6 \mathrm{H})$; ${ }^{13} \mathrm{C}$ NMR $\left(\mathrm{CDCl}_{3}\right)$ 2 214.6, 155.0, 80.4, 62.7, 54.2, 42.8, 37.4, 33.5, 32.1, 29.8, 29.4, 28.8, 26.2, 22.9, 22.8, 14.4. HRMS calcd for $\mathrm{C}_{20} \mathrm{H}_{37} \mathrm{NO}_{3}$ (M) 339.2773. Found 339.2764.

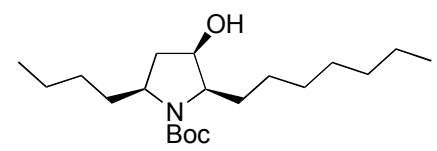

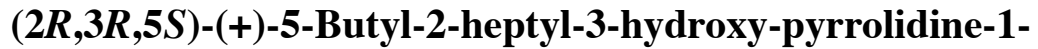
carboxylic acid tert-butyl ester (19). In a $25 \mathrm{~mL}$, singlenecked, round-bottom flask equipped with a magnetic stirring bar, rubber septum and argon balloon was placed (-)-18 $(0.126 \mathrm{~g}, 0.369 \mathrm{mmol})$ in $\mathrm{MeOH}(10 \mathrm{~mL})$. The solution was cooled to $0{ }^{\circ} \mathrm{C}$ and $\mathrm{NaBH}_{4}$ $(0.084 \mathrm{~g}, 2.21 \mathrm{mmol})$ was added. After stirring at $0{ }^{\circ} \mathrm{C}$ for $30 \mathrm{~min}$, the reaction mixture was quenched by addition of $\mathrm{H}_{2} \mathrm{O}(5 \mathrm{~mL})$. At this time the solution was extracted with EtOAc $(3 \times 10 \mathrm{~mL})$, the combined organic phases were washed with brine $(5 \mathrm{~mL})$, dried $\left(\mathrm{Na}_{2} \mathrm{SO}_{4}\right)$, and concentrated. Chromatography (hexanes:EtOAc, 9:1) gave $0.090 \mathrm{~g}(75 \%)$ 
of a colorless oil; $[\alpha]^{20}{ }_{\mathrm{D}}+19.8\left(c \mathrm{c} .8, \mathrm{CHCl}_{3}\right)$; IR (neat) $3465,2855,1685,1394 \mathrm{~cm}^{-1}$; ${ }^{1} \mathrm{H}$ NMR $\left(\mathrm{CDCl}_{3}\right)_{-} 4.28(\mathrm{~m}, 1 \mathrm{H}), 3.84(\mathrm{~m}, 1 \mathrm{H}), 3.67(\mathrm{~m}, 1 \mathrm{H}), 2.27(\mathrm{~m}, 1 \mathrm{H}), 1.97(\mathrm{~m}, 1$ $\mathrm{H}), 1.71(\mathrm{~m}, 1 \mathrm{H}), 1.61(\mathrm{~m}, 2 \mathrm{H}), 1.44(\mathrm{~s}, 9 \mathrm{H}), 1.41-1.20(\mathrm{~m}, 16 \mathrm{H}), 0.88(\mathrm{q}, J=9.2 \mathrm{~Hz}, 6$ $\mathrm{H}) ;{ }^{13} \mathrm{C} \mathrm{NMR}\left(\mathrm{CDCl}_{3}\right)_{-}$155.6, 79.5, 71.6, 60.8, 56.3, 37.9, 37.0, 32.2, 30.3, 30.0, 29.7, 28.8, 26.9, 23.0, 14.5, 14.4. HRMS calcd for $\mathrm{C}_{20} \mathrm{H}_{40} \mathrm{NO}_{3}(\mathrm{M}+\mathrm{H})$ 342.3008. Found 342.3007 .

(S,5R)-(-)-2-Butyl-5-heptyl-2,5-dihydro-pyrrole-1-carboxylic acid tert-butyl ester

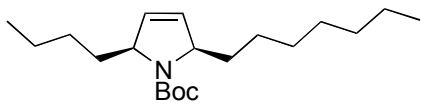

(20). In a 25-mL, single-necked, round-bottom flask equipped with a magnetic stirring bar, rubber septum, and argon balloon was placed (+)-19 $(0.090 \mathrm{~g}, 0.263 \mathrm{mmol})$ in

dichloromethane $(10 \mathrm{~mL})$. To the solution was added TEA $(0.074 \mathrm{~mL}, 0.526 \mathrm{mmol})$ and $\mathrm{MsCl}(0.041 \mathrm{~mL}, 0.526 \mathrm{mmol})$ at $\mathrm{rt}$. After stirring the reaction mixture for $2 \mathrm{~h}$, aqueous $\mathrm{NH}_{4} \mathrm{Cl}(5 \mathrm{~mL})$ was added to quench the reaction. The solution was extracted with DCM $(3 \times 10 \mathrm{~mL})$, the combined organic phases were washed with brine $(5 \mathrm{~mL})$, dried $\left(\mathrm{Na}_{2} \mathrm{SO}_{4}\right)$, and concentrated. The residue was dissolved in DMF $(15 \mathrm{~mL})$, the solution was cooled to $0{ }^{\circ} \mathrm{C}$ and $t$-BuOK $(1.0 \mathrm{M}$ in THF, $1.05 \mathrm{~mL}, 1.05 \mathrm{mmol})$ was added. At this time the reaction mixture was stirred at $\mathrm{rt}$ for $12 \mathrm{~h}$ and crushed ice $(5 \mathrm{~g})$ was added. The solution was extracted with $\mathrm{Et}_{2} \mathrm{O}(3 \times 25 \mathrm{~mL})$, the combined organic phases were washed with $\mathrm{H}_{2} \mathrm{O}(3 \times 10 \mathrm{~mL})$, dried $\left(\mathrm{Na}_{2} \mathrm{SO}_{4}\right)$, and concentrated. Chromatography (hexanes:EtOAc, 95:5) afforded $0.044 \mathrm{~g}(52 \%)$ of a colorless oil; $[\alpha]^{20}{ }_{\mathrm{D}}-6.4(c) 1.25$, $\left.\mathrm{CHCl}_{3}\right)$; IR (neat) 3334, 3026, 2921, $1689 \mathrm{~cm}^{-1} ;{ }^{1} \mathrm{H} \mathrm{NMR}\left(\mathrm{CDCl}_{3}\right) \_5.77(\mathrm{~m}, 1 \mathrm{H}), 5.71$ $(\mathrm{m}, 1 \mathrm{H}), 4.43(\mathrm{~m}, 1 \mathrm{H}), 4.34(\mathrm{~m}, 1 \mathrm{H}), 1.82(\mathrm{~m}, 2 \mathrm{H}), 1.47(\mathrm{~s}, 9 \mathrm{H}), 1.28(\mathrm{~m}, 16 \mathrm{H}), 0.88$ $(\mathrm{m}, 6 \mathrm{H}) ;{ }^{13} \mathrm{C} \mathrm{NMR}\left(\mathrm{CDCl}_{3}\right)_{-} 155.0,129.8,129.5,79.4,65.5,65.3,36.4,36.0,35.7,32.2$, 30.0, 29.7, 29.6, 28.9, 28.3, 28.2, 26.1, 26.0, 23.2, 23.1, 23.0, 14.4. HRMS calcd for $\mathrm{C}_{20} \mathrm{H}_{38} \mathrm{NO}_{2}(\mathrm{M}+\mathrm{H})$ 325.2902. Found 325.2904.

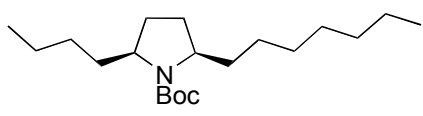

S(52R)-(-)-2-Butyl-5-heptyl-pyrrolidine-1-carboxylic acid tert-butyl ester (21). In a $25-\mathrm{mL}$, one-necked, roundbottomed flask equipped with a magnetic stirring bar, an outlet and inlet stopcock equipped with a $\mathrm{H}_{2}$-filled balloon and a rubber septum was placed $\mathrm{MeOH}(10 \mathrm{~mL}),(-)-20(0.044 \mathrm{~g}, 0.137 \mathrm{mmol})$, and Pt/C (ca. $0.020 \mathrm{~g}, 10 \mathrm{wt} \%$ on carbon). The reaction mixture was stirred for $30 \mathrm{~min}$ at $\mathrm{rt}$, the catalyst was removed by filtration through Celite, and the filtrate was concentrated. Chromatography (hexanes:EtOAc, 95:5) afforded $0.041 \mathrm{~g}(95 \%)$ of a colorless oil; $[\alpha]^{20}{ }_{\mathrm{D}}-1.2$ (c 1.2, $\left.\mathrm{CHCl}_{3}\right)$; IR (neat) 3334, 3026, $1668 \mathrm{~cm}^{-1} ;{ }^{1} \mathrm{H} \mathrm{NMR}\left(\mathrm{CDCl}_{3}\right)$ _ $3.72(\mathrm{~m}, 2 \mathrm{H}), 1.95-1.53$ $(\mathrm{m}, 6 \mathrm{H}), 1.45(\mathrm{~s}, 9 \mathrm{H}), 1.26(\mathrm{~m}, 16 \mathrm{H}), 0.88(\mathrm{t}, J=9.2 \mathrm{~Hz}, 6 \mathrm{H}) ;{ }^{13} \mathrm{C} \mathrm{NMR}\left(\mathrm{CDCl}_{3}\right)$ $155.4,79.0,58.7,36.4,36.0,32.2,30.0,29.7,29.0,28.9,26.8,23.1,23.0,14.5,14 . \overline{4}$. HRMS calcd for $\mathrm{C}_{20} \mathrm{H}_{40} \mathrm{NO}_{2}(\mathrm{M}+\mathrm{H})$ 326.3059. Found 326.3057.

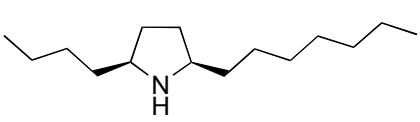

(2S,5R)-2-Butyl-5-heptyl-pyrrolidine (22). In a $25 \mathrm{~mL}$, single-necked, round-bottom flask equipped with a magnetic stirring bar, rubber septum, and argon balloon was placed ()-21 (0.041 g, $0.126 \mathrm{mmol})$ in dichloromethane $(4 \mathrm{~mL})$. The solution was cooled to $0{ }^{\circ} \mathrm{C}$ and trifluoroacetic acid $(1.0 \mathrm{~mL})$ was added. The ice bath was removed and the mixture was stirred for $1 \mathrm{~h}$ at $\mathrm{rt}$ before quenching with sat. $\mathrm{NaHCO}_{3}$ solution $(10 \mathrm{~mL})$. The 
solution was extracted with DCM $(3 \times 10 \mathrm{~mL})$, the combined organic phases were washed with brine $(5 \mathrm{~mL})$, dried $\left(\mathrm{Na}_{2} \mathrm{SO}_{4}\right)$, and concentrated. Chromatography (EtOAc) gave $0.025 \mathrm{~g}(88 \%)$ of a colorless oil; $[\alpha]^{20}{ }_{\mathrm{D}} 0\left(c 1.0, \mathrm{CH}_{3} \mathrm{OH}\right)$; IR (neat) $3300,2898 \mathrm{~cm}^{-1}$; ${ }^{1} \mathrm{H} \mathrm{NMR}\left(\mathrm{CDCl}_{3}\right) \_2.94(\mathrm{~m}, 2 \mathrm{H}), 2.87(\mathrm{~m}, 2 \mathrm{H}), 2.64(\mathrm{~m}, 2 \mathrm{H}), 1.27(\mathrm{~m}, 19 \mathrm{H}), 0.88(\mathrm{~m}$, $6 \mathrm{H}) ;{ }^{13} \mathrm{C}$ NMR $\left(\mathrm{CDCl}_{3}\right)_{-} 59.8,37.0,36.6,32.2,31.6,30.2,30.0,29.6,27.9,23.2,23.0$, 14.4. HRMS calcd for $\mathrm{C}_{15} \mathrm{H}_{32} \mathrm{~N}(\mathrm{M}+\mathrm{H})$ 226.2535. Found 226.2534.

\section{Reference}

(1) Davis, F. A.; Wu, Y.; Xu, H.; Zhang, J. Org. Lett. 2004, 6, 4523-4525 